\title{
Modelo predictivo de la distribución espacial de cobre en suelos agrícolas de la cuenca del Río Aconcagua, Chile
}

\author{
Cristina Hormazábal', labsuelo@ucv.cl; Rodrigo Aguilar'1, Marco Cisternas², \\ Alexander Neaman ${ }^{1}$
}

\section{RESUMEN}

Se presenta una propuesta metodológica que permitirá, sin necesidad de realizar un muestreo en terreno ni análisis de laboratorio, pronosticar preliminarmente la distribución del cobre en los suelos de una cuenca hidrológica. Esta información será de utilidad para posteriormente diseñar una estrategia de muestreo representativa y focalizada en áreas con potencialidad de contener el elemento en cuestión. Se espera que esta propuesta signifique finalmente un ahorro de recursos, tanto materiales como humanos. El modelo propuesto se fundamenta en la localización espacial y posterior clasificación de las eventuales fuentes contaminantes. El modelo fue sometido a prueba mediante el pronóstico de las concentraciones de cobre en los suelos agrícolas de la cuenca del Río Aconcagua. Finalmente, los resultados pronosticados fueron comparados con los resultados de un muestreo real efectuado en la cuenca. El pronóstico se validó en un $65 \%$ respecto a la distribución espacial y concentraciones de cobre observadas en la realidad.

Palabras clave: Sistema de Información Geográfica (SIG), minería, cobre, modelo predictivo.

\section{Predictive model of copper spatial distribution in agricultural soils of the Aconcagua river basin, Chile}

\begin{abstract}
A methodological proposal is presented, which will allow preliminary forecast the distribution of copper in the soils of a river basin, without conducting on-site sampling or laboratory analyses. This information will be useful for the subsequent design of a representative and focused sampling in areas with the potential of containing the element of interest. It is expected that this proposal will eventually lead to save resources, both material and human. The proposed model is based on the spatial positioning and subsequent classification of possible contaminating sources. The model was subject to a test through a forecast of copper concentrations in agricultural soils of the Aconcagua River basin. Finally, the forecasted results were compared to results of real sampling carried out at the basin. The forecast was validated in $65 \%$ regarding the spatial distribution of copper concentrations observed in reality.
\end{abstract}

Key words: Geographical Information System (GIS), mining, copper, predictive model.

Recibido el 4 de julio de 2013, aceptado el 11 de noviembre de 2013.

\footnotetext{
1 Área del Medio Ambiente, Facultad de Agronomía, Pontificia Universidad Católica de Valparaíso, Chile. San Francisco s/n, La Palma, Quillota, Región de Valparaíso, Chile.

2 Escuela de Ciencias del Mar, Pontificia Universidad Católica de Valparaíso, Chile.
} 


\section{INTRODUCCIÓN}

El cobre es importante contaminante ambiental asociado principalmente a las actividades mineras (DE GREGORI et al. 2003). Éste altera las características naturales de los suelos en sectores cercanos a dichas actividades y pueden provocar efectos adversos sobre los organismos vivos del suelo y sobre los cultivos agrícolas. El cobre puede ser tóxico para todos los organismos dependiendo de las concentraciones en que se encuentren (ADRIANO 2001). En este sentido, la agricultura y la minería son actividades conflictivas en Chile (GONZÁLEZ 1991).

La cuenca del río Aconcagua (Fig. 1), con $7.334 \mathrm{~km}^{2}\left(32^{\circ} 20^{\prime}-33^{\circ} 07^{\prime} \mathrm{S}\right.$ y $71^{\circ} 31^{\prime}$ - $\left.70^{\circ} 00^{\prime} \mathrm{N}\right)$, se caracteriza por contar con una importante población (casi medio millón de habitantes) y diversidad de actividades económicas basadas en sus recursos naturales (INSTITUTO NACIONAL DE ESTADÍSTICAS 2005). Entre estas últimas destacan la minería de cobre y la agricultura intensiva (DIRECCIÓN GENERAL DE AGUAS 2004a y 2004b). La minería está representada por 40 minas, 13 plantas (lixiviación y concentración) $\mathrm{y}$ una fundición (LARA \& ROMO, 2002). Por su parte, la agricultura se caracteriza por desarrollar una actividad intensiva, con riego, orientada tanto al mercado interno como a la exportación (COMISIÓN NACIONAL DE RIEGO 2000; DIRECCIÓN GENERAL DE AGUAS 2004b).

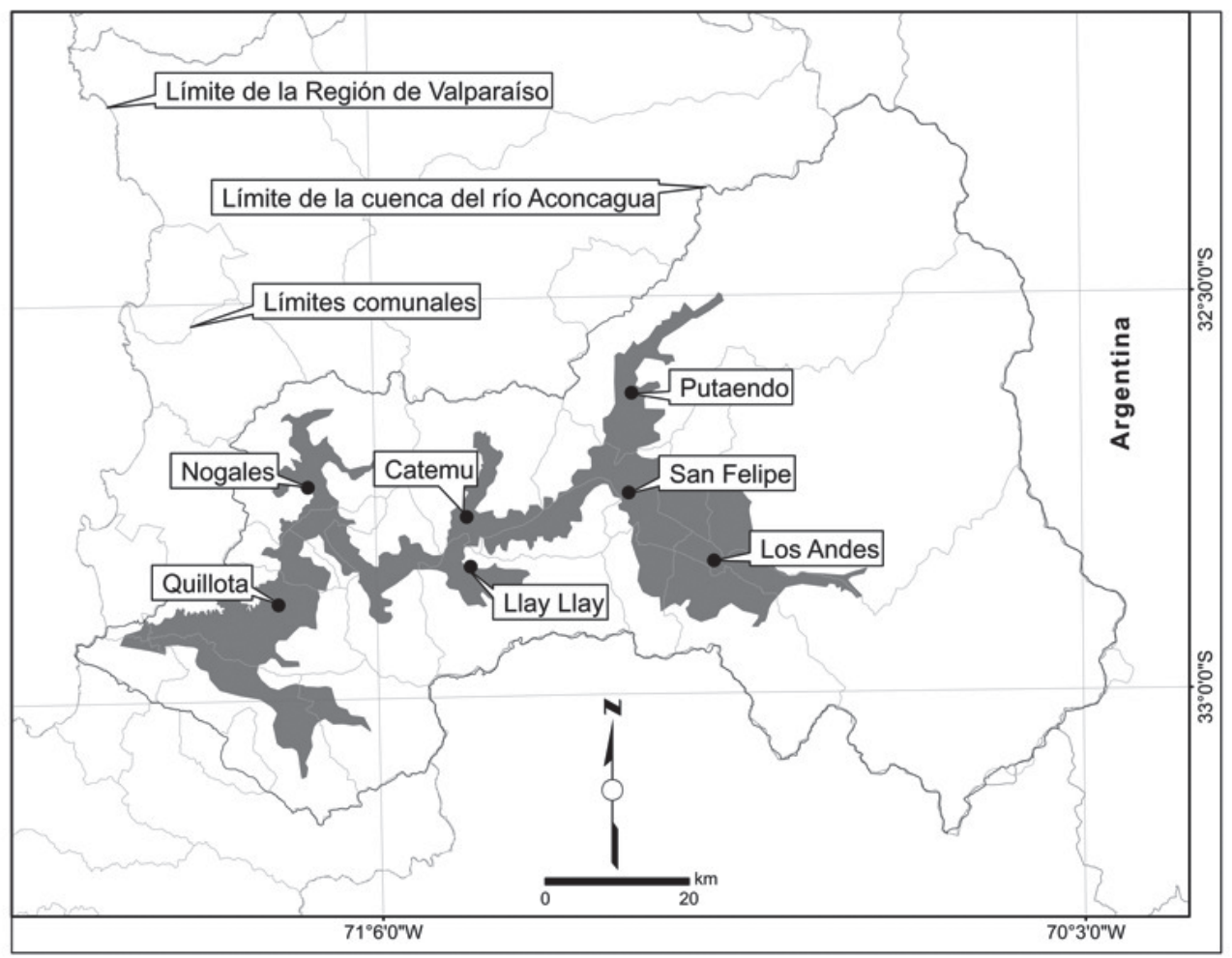

Fig. 1. Ubicación geográfica del área de estudio, que consideró suelos con pendientes menores al $15 \%$ (área gris). Fuente: Elaborado por los autores.

Fig. 1. Geographical location of the study area, which considered soils with slopes less than $15 \%$ (gray area). Source: Elaborated by the authors. 
Considerando lo anterior, es importante, como un primer paso en la evaluación de los efectos de la minería del cobre sobre la producción agrícola, conocer la distribución geográfica de cobre en los suelos que actualmente están siendo utilizados por la agricultura. Paralelamente, también es necesario determinar si hay una relación espacial entre las actividades mineras y las concentraciones de cobre en los suelos agrícolas.

Debido al alto costo de realizar muestreos y análisis de superficies extensas, como lo es una cuenca hidrográfica, el desarrollo de cualquier metodología, directa o indirecta, que permita una aproximación a la distribución del cobre en la cuenca del Río Aconcagua facilitará la toma de decisiones a la hora de diseñar y ejecutar muestreos y programas de análisis. En este sentido, se plantea que el análisis de información de base, mediante un Sistema de Información
Geográfica (SIG), en conjunto con programas de ponderación de variables, permitirá pronosticar la distribución y concentración relativa del cobre en los suelos de la cuenca. Así, el presente trabajo propone un modelo para predecir la distribución espacial de las concentraciones de cobre en suelos agrícolas potencialmente afectados por actividades mineras.

\section{MATERIALES Y MÉTODOS}

\section{Construcción del modelo}

El modelo fue construido sobre la base de cuatro grandes etapas (Fig. 2): i) delimitación del área de estudio; ii) recopilación de información (base de datos); iii) determinación y análisis de los atributos de impacto de las fuentes (ponderación y suma lineal); y iv) obtención de la información espacial y del pronóstico.

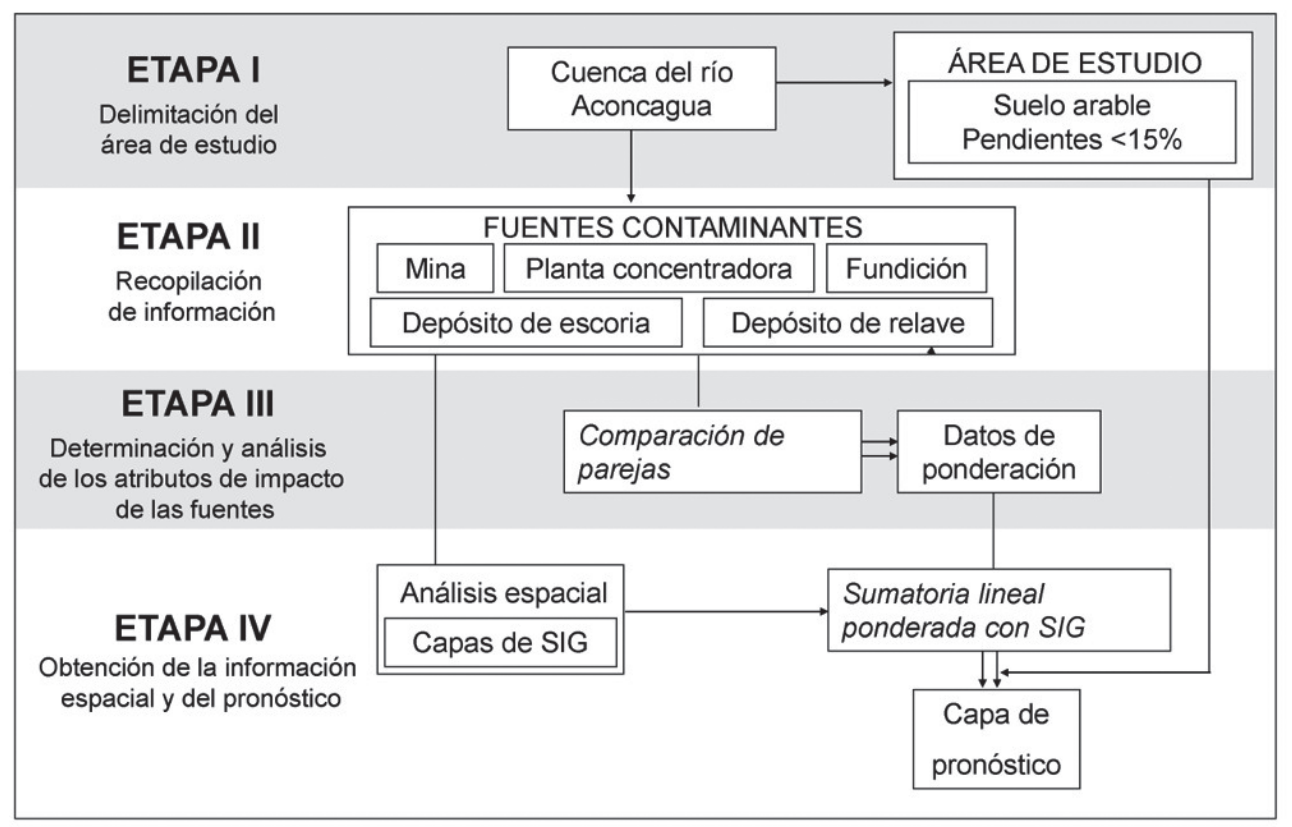

Fig. 2. Esquema del modelo de pronóstico. Fuente: Elaborado por los autores.

Fig. 2. Scheme of the predictive model. Source: Elaborated by the authors. 


\section{Etapa I. Delimitación del área de estudio}

Se consideró como área de estudio la cuenca del Río Aconcagua, a modo de unidad territorial contenedora tanto de las fuentes potencialmente contaminantes de cobre (minas, plantas concentradoras, fundición, depósitos de relaves y depósitos de escorias), como de los suelos agrícolas potencialmente afectados. La cuenca hidrográfica se delimitó a través de la topografía, identificando la divisoria de aguas (escala 1:50.000). Para la delimitación de los suelos tradicionalmente utilizados en la agricultura se utilizaron dos criterios: localización respecto a la cota de canal y pendiente. De este modo, se consideraron todos los suelos que están bajo la cota de canal, es decir, que pueden ser regados de modo gravitacional y con pendientes menores al $15 \%$ (Fig. 1, área gris).

\section{Etapa II. Recopilación de información}

Se identificó y digitalizó la información geográfica de base que permitió la caracterización de la cuenca (escala 1:50.000). Esto es, límites administrativos, ríos, caminos, curvas de nivel y red de canales (COMISIÓN NACIONAL DE RIEGO 2000). Sobre la base de la información bibliográfica, se identificaron las fuentes potencialmente contaminantes de cobre al interior de la cuenca del Río Aconcagua (LARA \& ROMO 2002; ARANCIBIA 2002). Se determinaron cinco tipos de fuentes contaminantes según la actividad que realizan: mina, planta concentradora, fundición, depósito de relave, y depósito de escoria. Al no existir información previa publicada relativa a los depósitos de escoria, fue necesario realizar un reconocimiento en terreno para su identificación. La localización de la mayoría de las fuentes fue obtenida a través de las coordenadas geográficas publicadas, mientras que una minoría fueron georeferenciadas con GPS durante el trabajo de campo.
Etapa III. Determinación y análisis de los atributos de impacto de las fuentes

El primer paso para llegar a pronosticar, mediante el modelo, la concentración de cobre en los suelos agrícolas, fue ponderar la influencia que tiene cada fuente reconocida sobre la distribución espacial de cobre en la cuenca. Para esto, fue necesario asignarles, a cada una, atributos de impacto cuantificable numéricamente. Considerando la diversidad de las fuentes analizadas, se establecieron cuatro grandes tipos de atributos, estando los tres primeros en acuerdo con la clasificación de LARA \& ROMO (2002), a saber,

1) tamaño: se refiere al tamaño de la actividad, variando desde la categoría $\mathrm{A}$, la más grande, hasta la $\mathrm{D}$, la más pequeña

2) estado: se refiere a la condición de funcionamiento, es decir, si está paralizada o en actividad 3) proceso: se refiere a la relación con la diversidad de sus procesos productivos

4) extensión: se refiere a la amplitud geográfica de su influencia ambiental, determinada por las características físicas del sistema natural, esencialmente la dirección del viento y del drenaje de aguas superficiales.

En base a la opinión de expertos (los coautores del artículo y experto, Ingeniero Civil de Minas, Servicio Nacional de Geología y Minería), se asignaron atributos específicos a cada tipo de fuente (Tabla 1). Así, por ejemplo, el atributo proceso fue asignado sólo a minas y plantas, pero no a las restantes fuentes. Posteriormente cada fuente fue jerarquizada respecto a sus similares, utilizando los atributos asignados. 
Tabla 1. Atributos de impacto asignados a cada fuente. Fuente: Elaborado por los autores

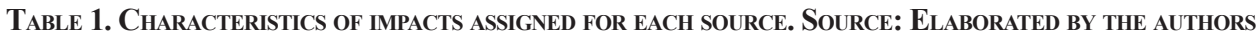

\begin{tabular}{|c|c|c|c|c|c|}
\hline $\begin{array}{r}\text { Fuente } \\
\text { Atributo }\end{array}$ & Minas & Plantas concentradoras & Fundición & Depósito de relave & Depósito de escoria \\
\hline Tamaño & $\mathrm{X}$ & $\mathrm{X}$ & $\mathrm{X}$ & & \\
\hline Estado & $\mathrm{X}$ & $\mathrm{X}$ & $\mathrm{X}$ & $\mathrm{X}$ & $\mathrm{X}$ \\
\hline Proceso & $\mathrm{X}$ & $\mathrm{X}$ & & & \\
\hline Extensión & $\mathrm{X}$ & $\mathrm{X}$ & $\mathrm{X}$ & $\mathrm{X}$ & $\mathrm{X}$ \\
\hline
\end{tabular}

El atributo tamaño fue asignado a las minas, plantas concentradoras y fundición debido a que existe una categorización previamente establecida que considera los volúmenes procesados por cada una de estas tres fuentes y el número de sus operarios (LARA \& ROMO 2002; FOLCHI 2006). Así es posible fundamentar su jerarquización sobre parámetros cuantitativos.

El atributo estado se asignó a todas las fuentes pues existe suficiente información previa respecto al estado de actividad de las minas y plantas concentradoras (LARA \& ROMO 2002) y de los depósitos de relave (ARANCIBIA 2002). De las 39 minas presentes en la cuenca, sólo 12 se encuentran activas y 27 paralizadas. De las 13 plantas concentradoras, 7 están en pleno funcionamiento y 6 paralizadas. De los 14 depósitos de relave, 12 se encuentran activos y sólo 2 paralizados. De las observaciones en terreno, se revela que de los tres depósitos de escorias, el que pertenece a la Fundición Chagres se encuentra activo, mientras que otros dos, ubicados ambos en Nilhue, estaban paralizados.

El atributo proceso fue asignado sólo a minas y plantas concentradoras debido a que ambas presentan gran variabilidad en sus labores (LARA \& ROMO 2002). En cambio, las restantes fuentes presentan homogeneidad entre ellas. Del total de minas analizadas, 3 extraen el mineral de cobre mediante rajo abierto, 35 con perforaciones subterráneas y una mezcla ambos sistemas. De las 13 plantas concentradoras de cobre en la cuenca, 8 utilizan el proceso de concentración y 5 el de lixiviación (LARA \& ROMO 2002). El panel de expertos consideró que las plantas de lixiviación presentan mayor impacto, en comparación con las de concentración (Fig. 3).

Debido a la importancia del atributo extensión, éste fue asignado a todas las fuentes. Para determinarlo, se utilizó el sentido de la escorrentía superficial (red hídrica a escala de microcuencas), asumiendo que el principal medio exportador de contaminantes (líquidos y sólidos) desde la fuente es la escorrentía superficial. Esta aproximación se utilizó en las minas, plantas concentradoras, depósitos de relave $\mathrm{y}$ de escoria. En el caso de las emisiones aéreas de la única fundición en la cuenca (Fundición Chagres), la variable considerada fue la dirección y frecuencia de los vientos, como principal mecanismo controlador de la distribución de contaminantes desde la chimenea de la fundición.

Finalmente, para poder pronosticar la distribución de cobre en la cuenca, se procedió a ponderar las características de los atributos y de las fuentes. La ponderación se realizó en base al Método de Comparación de Parejas. En este método, el primer paso es realizar una comparación verbal de las características de los atributos de acuerdo a la escala del Tabla 2 (obtenido desde el software Expert Choice v. 2000). Dicha escala permite comparar linealmente la importancia de un atributo respecto al otro. 
Tabla 2. Escala de comparaciones. Fuente: Software EXPERT CHOICE v. 2000

Table 2. Scale of Comparisons. Source: Software EXPERT CHOICE v. 2000

\begin{tabular}{|c|c|}
\hline Valor numérico & Escala verbal \\
\hline 1 & Igual importancia de ambos atributos \\
\hline 3 & Moderada importancia de un atributo frente a otro \\
\hline 5 & Fuerte importancia de un atributo sobre otro \\
\hline 7 & Muy fuerte importancia de un atributo sobre otro \\
\hline 9 & Extrema importancia de un atributo sobre otro \\
\hline
\end{tabular}

En un segundo paso, los datos obtenidos de la comparación verbal son ingresados al programa de ponderación Expert Choice, el que finalmente entrega un resultado numérico. Estos valores permiten jerarquizar y analizar tanto los atributos (Fig. 3) como las fuentes (Fig. 4) mediante el SIG.

Finalmente, con el objetivo de validar las comparaciones, se utilizó la aproximación de BARREDO (1996). Este autor propone que para chequear la consistencia de los valores de comparación, se debe utilizar el factor denominado razón de consistencia ( $\mathrm{CR}$, consistency ratio). Si CR es mayor o igual a 0,1 , se demuestra que los juicios de valor no son lo suficientemente consistentes para la posterior ponderación; por el contrario, si CR es $<0,1$, los juicios de valor asignados son satisfactorios.
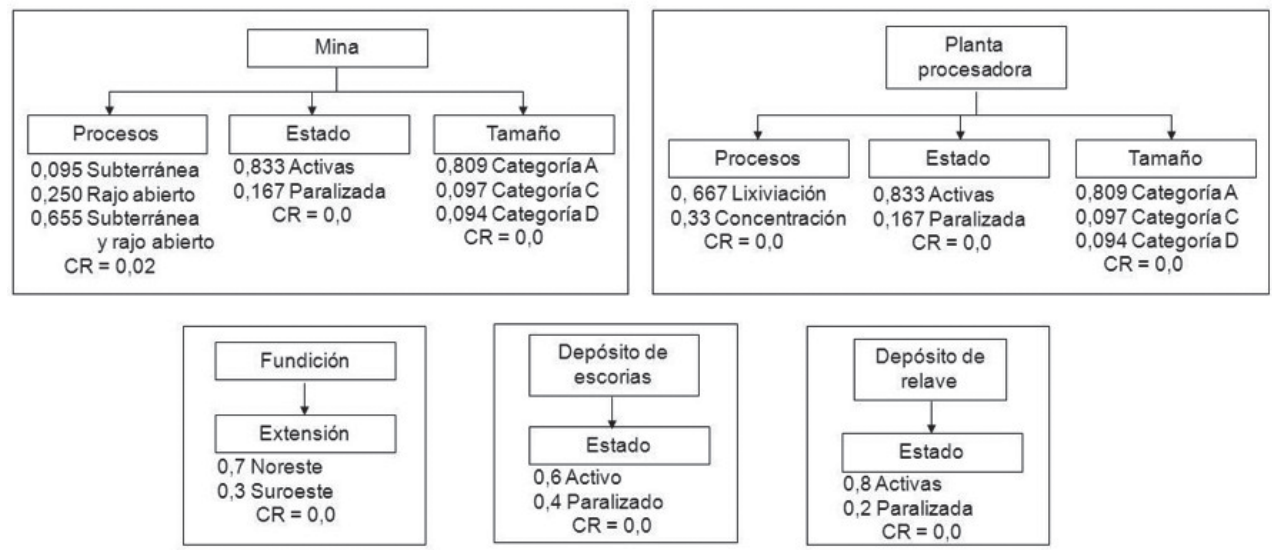

Fig. 3. Ponderación de los atributos de las fuentes contaminantes. Fuente: Elaborado por los autores.

Fig. 3. Weighting of the characteristics of contaminating sources. Source: Elaborated by the authors. 


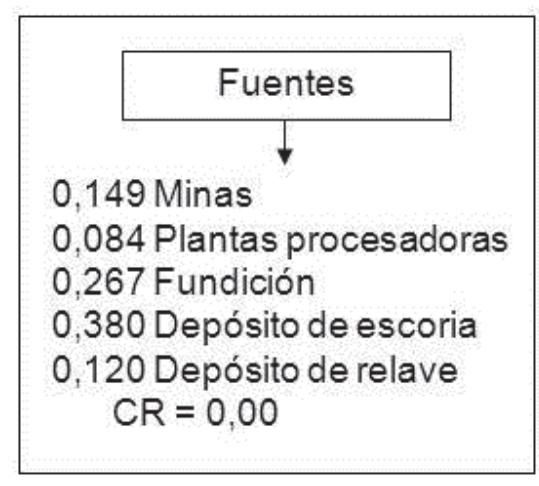

Fig. 4. Ponderación de las fuentes contaminantes. Fuente: Elaborado por los autores.

Fig. 4. Weighting of the contaminating sources. Source: Elaborated by the authors.

Etapa IV. Obtención de la información espacial y del pronóstico

El análisis espacial de la información se realizó considerando la distancia lineal desde las fuentes contaminantes y su sistema de drenaje superficial. Localizando a la fuente en el centro, se trazaron áreas concéntricas que representan su nivel de influencia espacial. Las distancias radiales fueron establecidas a los 2, 5, 10, 20, 30, 40 y $50 \mathrm{~km}$. De este modo, siguiendo lo propuesto por BARREDO (1996), el área adyacente a la fuente presenta el máximo valor normalizado (255), mientras que el área distante a $50 \mathrm{~km}$ representa la menor influencia (0). Considerando que el drenaje superficial es uno de los principales agentes de dispersión de contaminantes, se delimitaron las microcuencas en que están insertas cada una de las fuentes. Finalmente, superponiendo ambas áreas, las concéntricas y las de drenaje, se obtuvieron las áreas de contaminación potencial.

Con el objetivo de determinar el grado de contaminación potencial, las áreas fueron ponderadas de acuerdo a los atributos específicos de cada fuente, generándose capas de información. En el caso de las minas y plantas concentradoras, se diferenciaron entre activas y paralizadas.
Para las minas activas, se obtuvieron tres capas de información de acuerdo a sus procesos: subterráneo, rajo abierto y la combinación de ambos. En el caso de las minas paralizadas, todas son subterráneas, por lo que se obtuvo sólo una capa de información. Respecto a las plantas concentradoras, se obtuvieron dos capas para el atributo activas y dos para las paralizadas, en ambos casos corresponden a los procesos de lixiviación y concentración. El atributo tamaño generó tres capas que corresponden a las tres categorías (A, C y D), tanto para minas como para plantas. Tanto para los depósitos de relave como para los depósitos de escoria se obtuvieron dos capas (estado activo o paralizado). Para la fuente fundición se obtuvieron dos capas de información de acuerdo a la dirección y predominancia del viento (NE y SW).

Posteriormente, mediante la sumatoria de las diferentes capas de información, se obtuvieron sólo cinco capas, cada una representando a una fuente. Finalmente, se realizó una sumatoria lineal ponderada de las cinco capas logrando definir la superficie y el grado de la contaminación potencial en el área de estudio.

\section{RESULTADOS}

\section{Pronóstico del modelo}

Para representar cartográficamente tanto los resultados del modelo (capa pronóstico), como los de su posterior validación (ver más abajo), se determinaron rangos de valor, mediante el criterio de umbrales naturales o Natural Breaks, que ofrece el ArcGIS versión 9.2 (Tabla 3). Este método identifica saltos importantes en la secuencia de valores para crear clases o rangos, a través de la aplicación de una fórmula estadística de George Jenks, que minimiza la variación entre cada clase. Además permite ver agrupaciones y patrones de distribución inherentes a los datos (ENVIROMENTAL SYSTEMS RESEARCH INSTITUTE 
1996; FISCHER 1999). Los rangos de umbrales naturales fueron clasificados, en forma cualitativa, como bajo, medio y alto.

Tabla 3. UMbrales naturales de las CONCENTRACIONES PRONOSTICADAS, UNIDADES relativas de 0 a 255. Fuente: Elaborado por LOS AUTORES

Table 3. Natural breaks of the Predicted CONCENTRATIONS, RELATIVE UNITS FROM 0 TO 255. SOURCE: Elaborated BY THE AUTHORS

\begin{tabular}{|c|c|}
\hline $\begin{array}{c}\text { Pronóstico } \\
\text { (unidades relativas de 0 a 255) }\end{array}$ & $\begin{array}{c}\text { Clasificación } \\
\text { cualitativa }\end{array}$ \\
\hline$<31,94$ & Bajo \\
\hline $31,94-78,33$ & Medio \\
\hline$>78,33$ & Alto \\
\hline
\end{tabular}

De este modo, el modelo fue capaz de pronosticar las concentraciones potenciales de cobre sobre un área de $1153 \mathrm{~km}^{2}$ de suelos agrícolas (Fig. 5), que corresponde a la totalidad del área de estudio. En general, el modelo pronostica bajas concentraciones de cobre en la mayor parte del área, sobre el $89 \%$ de la superficie total, que corresponde a unos $1000 \mathrm{~km}^{2}$. Estas concentraciones se localizarían principalmente en los extremos oriental y occidental, es decir, en la parte alta y baja de la cuenca, respectivamente.

Las concentraciones medias se pronostican en el $9 \%$ de la superficie total, correspondiendo a $106 \mathrm{~km}^{2}$, principalmente localizados en la zona central del área de estudio a modo de tres parches (Fig. 5). El parche más grande rodearía a un núcleo de concentración alta ubicado justo en el centro del área de estudio, mientras que el parche occidental y el oriental se asociarían a zonas de importante actividad minera (i.e. embalses, minas, depósitos de escorias).

Las mayores concentraciones pronosticadas sólo cubren un $2 \%$ del área, abarcando $23 \mathrm{~km}^{2}$. Sin embargo, su distribución geográfica se observa altamente concentrada en un núcleo, más o menos circular, en el centro del área de estudio, alrededor de la Fundición Chagres.

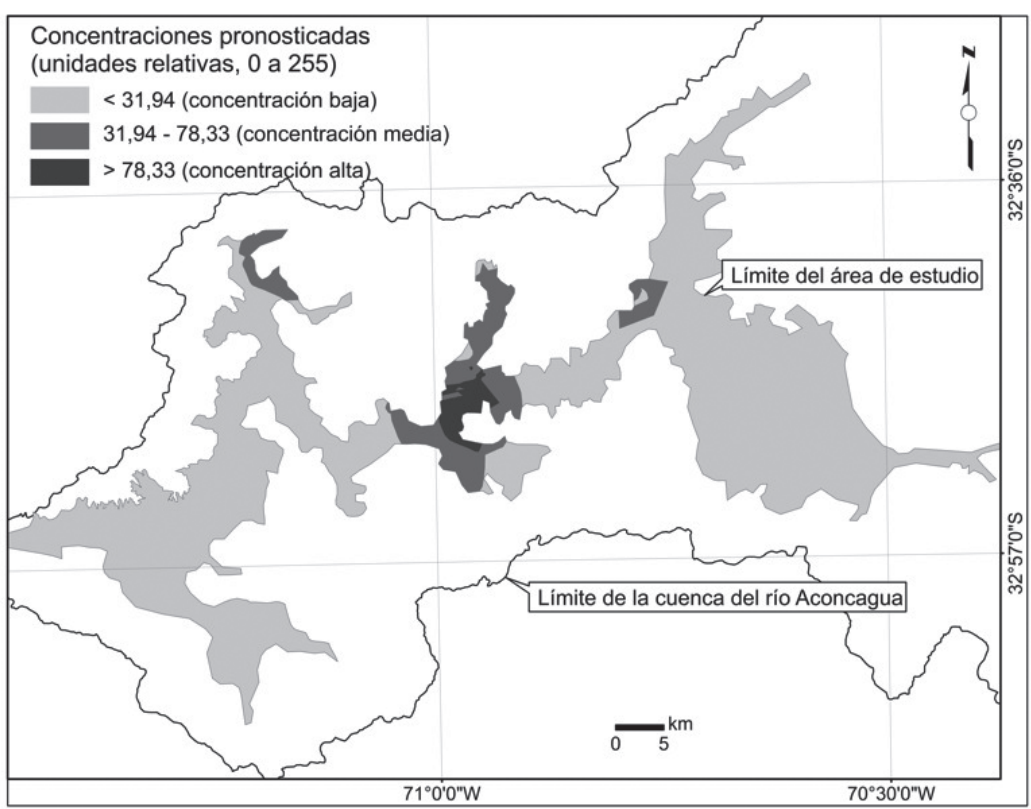

Fig. 5. Pronóstico de concentraciones de cobre en suelos agrícolas, de acuerdo al modelo propuesto (unidades relativas de 0 a 255). Fuente: Elaborado por los autores.

Fig. 5. Prognostics of copper concentrations in agricultural soils, according to the proposed model (relative units from 0 to 255). Source: Elaborated by the authors. 


\section{Validación del modelo}

Con el objetivo de validar el modelo, se comparó sus pronósticos con la distribución real de cobre en los suelos agrícolas de la cuenca del río Aconcagua (Fig. 6). En el 2005, se realizó un extensivo muestreo de los suelos de la cuenca para determinar su concentración de cobre total en más de 100 muestras (NEAMAN et al. 2006;
AGUILAR et al. 2011). Los detalles del protocolo de muestreo se presenta en AGUILAR etal.(2011). Utilizando los datos de concentración de aquella investigación, se hizo una representación cartográfica de la distribución espacial del cobre mediante la interpolación de los valores obtenidos en cada punto de muestreo (capa realidad). Esto generó una serie de áreas semicirculares de $2 \mathrm{~km}$ de diámetro (Fig. 7).

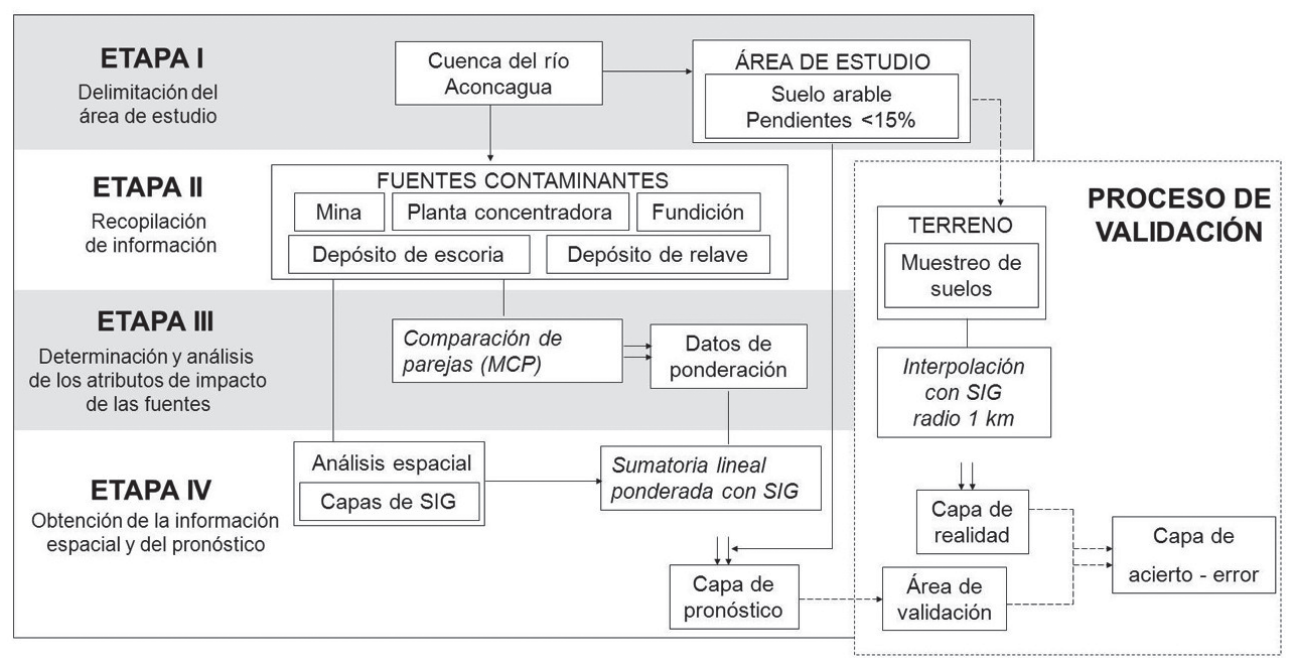

Fig. 6. Proceso de validación del modelo. Fuente: Elaborado por los autores.

Fig. 6. Process of model validation. Source: Elaborated by the authors.

Los rangos de concentración se determinaron, al igual que en el caso del pronóstico, con el criterio de umbrales naturales (Tabla 4). Estos rangos de umbrales naturales fueron clasificados, en forma cualitativa, como bajo, medio y alto y no representan juicio sobre el potencial riesgo ecotoxicológico de cobre en los suelos estudiados.
Tabla 4. Umbrales naturales de las Concentraciones reales. Fuente: Elaborado POR LOS AUTORES

Table 4. Natural breaks of real Concentrations. Source: Elaborated by THE AUTHORS

\begin{tabular}{|c|c|}
\hline $\begin{array}{c}\text { Real } \\
(\mathrm{mg} / \mathrm{kg})\end{array}$ & Clasificación cualitativa \\
\hline$<382,17$ & Bajo \\
\hline $382,17-1495,21$ & Medio \\
\hline$>1495,21$ & Alto \\
\hline
\end{tabular}




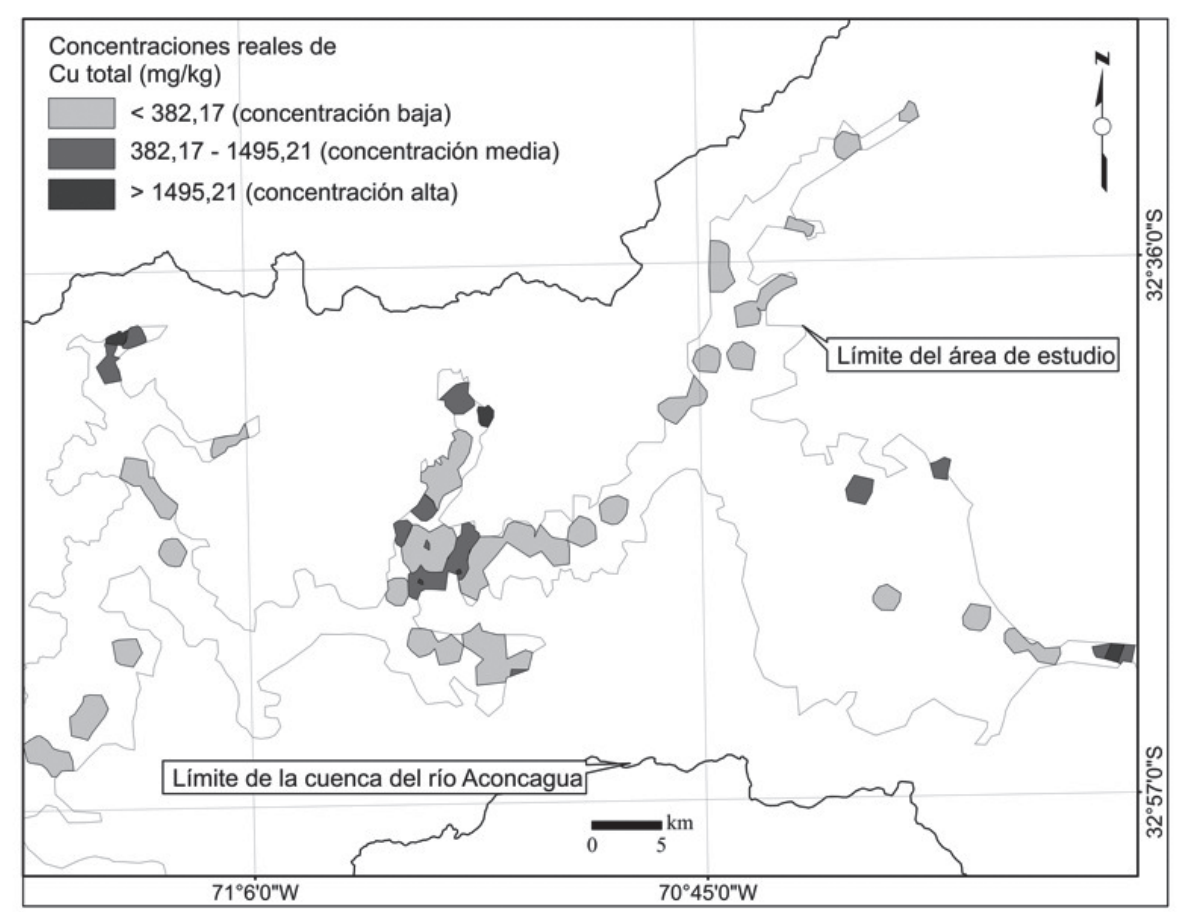

Fig. 7. Las concentraciones reales de cobre, en base a los estudios de NEAMAN et al. (2006) y AGUILAR et al. (2011). Fuente: Elaborado por los autores.

Fig. 7. Real copper concentrations, based on the studies of NEAMAN et al. (2006) y AGUILAR et al. (2011). Source: Elaborated by the authors.

Con el objetivo de determinar el grado de acierto o error del pronóstico, las capas pronóstico y realidad fueron intersectadas entre ellas. Para lograr esto, fue necesario previamente reclasificar sus rangos (alto, medio, bajo) a valores ordinales $(3,2$, 1). La intersección generó una tercera representación cartográfica que muestra las áreas donde el pronóstico obtuvo acierto, error menor o error mayor (capa acierto-error; Fig. 8). Se consideró que el pronóstico del modelo tuvo acierto cuando coincidió con la clasificación de la realidad, por ejemplo alto-alto. En cambio, el modelo tuvo un error menor cuando a pesar de no ser coincidente, el pronóstico correspondía, en realidad, a la clasificación inmediatamente adyacente, como por ejemplo alto-medio. El modelo presentó un error mayor cuando el pronóstico fue totalmente opuesto al de la realidad, por ejemplo alto-bajo. Se obtuvo un $65 \%$ de acierto en el pronóstico de la distribución (Fig. 8). 


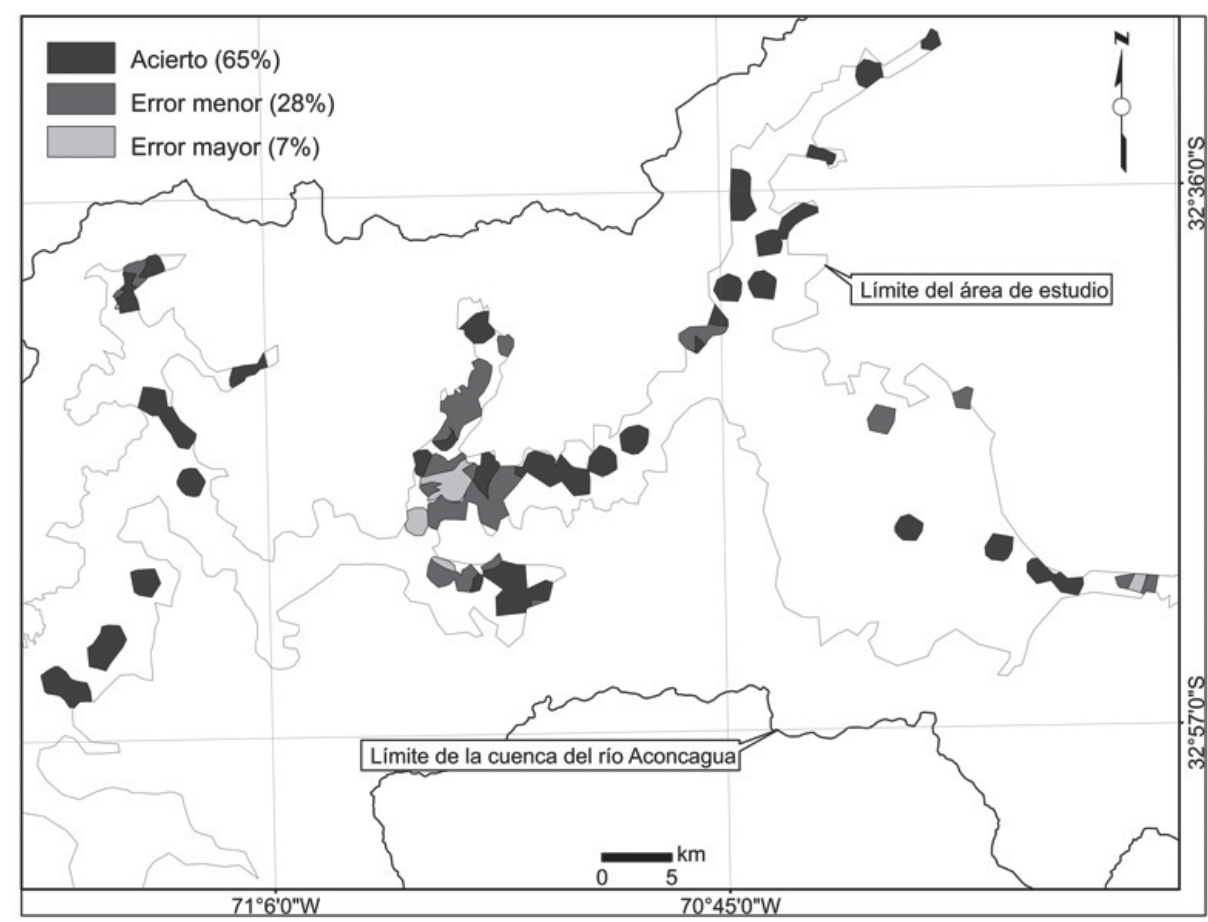

Fig. 8. Acierto y error del pronóstico, respecto a la realidad. Fuente: Elaborado por los autores.

Fig. 8. Correct and wrong prediction, with respect to the reality. Source: Elaborated by the authors.

\section{DISCUSIÓN}

La Tabla 5 presenta probables causas de errores en pronóstico del modelo, en cada una de las zonas de estudio. El análisis de los resultados reveló la correcta predicción de la zona Quillota, al ser correctamente valorada la ausencia de mineras activas, lo que explicaría su acierto total (100\%).

El error en la zona El Melón se atribuye en gran medida a los estragos dejados por el terremoto de 1965. Este suceso provocó la ruptura de un depósito de relave afectando un área de $8 \mathrm{~km}$ hasta las cercanías de El Melón (FOLCHI 2003). Lo anterior se vio reflejado al analizar la distribución que presentaban las concentraciones del muestreo real.

En Ñilhue, se sobrestimó la antigüedad de los depósitos de escorias, al asumir que éstos no estaban activos. Estos depósitos se asocian a dos fundiciones que existieron a partir del año 1841 y en el comienzo del siglo XX (HUIDOBRO 1861; FOLCHI, 2006).

En la zona de Catemu, los resultados se mostraron bastante heterogéneos en las cercanías de la Fundición Chagres, lo que se explicaría por el comportamiento heterogéneo del viento. Lo anterior afecta la orientación de la pluma de la chimenea de la fundición, influyendo sobre la distribución de material particulado (GONZÁLEZ \& ITE 1992). Estos resultados son similares a los de GINOCCHIO et al. (2004) que reportaron una distribución heterogénea de las concentraciones de cobre en las cercanías de la Fundición Ventanas. A pesar de los actuales manejos realizados por la fundición, hay que tener presente que las acciones mitigantes de sus emisiones atmosféricas se iniciaron en 1991, lo que explicaría en parte la heterogénea 
concentración encontrada (FOLCHI 2006). De acuerdo a lo analizado por BADILLAOHLBAUM et al. (2001), los elementos minerales presentes en los depósitos de relave pueden ser arrastrados por ríos. Esto afectó aún más la heterogeneidad de los resultados en Catemu.

En la zona de Catemu, los resultados se mostraron bastante heterogéneos en las cercanías de la Fundición Chagres, lo que se explicaría por el comportamiento heterogéneo del viento. Lo anterior afecta la orientación de la pluma de la chimenea de la fundición, influyendo sobre la distribución de material particulado (GONZÁLEZ \& ITE 1992). Estos resultados son similares a los de GINOCCHIO et al. (2004) que reportaron una distribución heterogénea de las concentraciones de cobre en las cercanías de la Fundición Ventanas. A pesar de los actuales manejos realizados por la fundición, hay que tener presente que las acciones mitigantes de sus emisiones atmosféricas se iniciaron en 1991, lo que explicaría en parte la heterogénea concentración encontrada (FOLCHI 2006). De acuerdo a lo analizado por BADILLAOHLBAUM et al. (2001), los elementos minerales presentes en los depósitos de relave pueden ser arrastrados por ríos. Esto afectó aún más la heterogeneidad de los resultados en Catemu.

El porcentaje de error obtenido en Llay Llay se atribuye en parte al efecto de arrastre de escorias provenientes de los depósitos de Chagres, ya que en la zona más grande de error no existiría fuente alguna de cobre como minas o tranques.

En la zona de Putaendo, el alto porcentaje de acierto se establecería por las categorías de las minas, las cuales pertenecen a la pequeña minería. SÁNCHEZ \& ENRÍQUEZ (1996) reportaron un bajo impacto ambiental en la calidad de suelo y agua afectado por pequeñas mineras.

En Los Andes, el error presentado se atribuiría al arrastre de partículas a través del río. Aunque el complejo minero de CODELCO, División Andina, se presenta como una posible fuente de cobre en este sector, es necesario realizar más estudios para lograr establecer un patrón en la distribución de este elemento entre el río y la actividad minera.

Tabla 5. Probables causas de errores en pronóstico del modelo, en cada una de las zonas de ESTUdio. Fuente: Elaborado POR LOS AUTORES

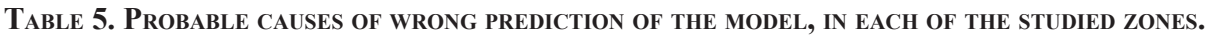
Source: Elaborated by the aUthors

\begin{tabular}{|c|c|c|c|c|c|}
\hline Localidad & Acierto (\%) & $\begin{array}{c}\text { Error } \\
\text { menor (\%) }\end{array}$ & $\begin{array}{c}\text { Error } \\
\text { mayor (\%) }\end{array}$ & Tipo del error & Detalles \\
\hline El Melón & 45 & 36 & 18 & $\begin{array}{c}\text { Similitud de } \\
\text { error por sub y } \\
\text { sobrestimación }\end{array}$ & $\begin{array}{c}\text { Subestimación del } \\
\text { manejo ambiental de los } \\
\text { depósitos de relave luego } \\
\text { del terremoto. } \\
\text { Sobrestimación de gran } \\
\text { minería presente. }\end{array}$ \\
\hline Nilhue & 47 & 42 & 11 & Sobreestimación & $\begin{array}{r}\text { El error mayor obtenido } \\
\text { se atribuye a la } \\
\text { sobrestimación del } \\
\text { impacto de depósitos } \\
\text { de escoria. }\end{array}$ \\
\hline
\end{tabular}




\begin{tabular}{|c|c|c|c|c|c|}
\hline Localidad & Acierto (\%) & $\begin{array}{c}\text { Error } \\
\text { menor (\%) }\end{array}$ & $\begin{array}{c}\text { Error } \\
\text { mayor (\%) }\end{array}$ & Tipo del error & Detalles \\
\hline Catemu & 40 & 32 & 28 & $\begin{array}{c}\text { Sector } \\
\text { heterogéneo }\end{array}$ & $\begin{array}{c}\text { La chimenea de la } \\
\text { fundición Chagres } \\
\text { produce concentraciones } \\
\text { heterogéneas en el suelo. }\end{array}$ \\
\hline Llay Llay & 25 & 63 & 13 & Sobreestimación & $\begin{array}{c}\text { Sobrestimación del efecto } \\
\text { de los depósitos de } \\
\text { escorias de la fundición } \\
\text { Chagres. }\end{array}$ \\
\hline Putaendo & 92 & 8 & 0 & Subestimación & $\begin{array}{c}\text { Subestimación del } \\
\text { impacto del depósito } \\
\text { de relave de la mina } \\
\text { Bellavista. }\end{array}$ \\
\hline Andes & 43 & 43 & 14 & Subestimación & $\begin{array}{c}\text { Se subestimo el impacto } \\
\text { del complejo minero de } \\
\text { CoDELCO, División }\end{array}$ \\
\hline
\end{tabular}

\section{CONCLUSIONES}

El método propuesto fue efectivo en un $65 \%$ para pronosticar la distribución de cobre en suelos agrícolas de la cuenca del río Aconcagua. Esto se logró realizando el mínimo de visitas a terreno y sin realizar ningún análisis de terreno de las variables incluidas en la metodología. El alto porcentaje de acierto se expresaría como el ahorro que se produce al guiar un muestreo para determinar una distribución espacial de metales en áreas mineras.

\section{REFERENCIAS}

ADRIANO, D.C., 2001. Trace Elements in Terrestrial Environments: Biogeochemistry, Bioavailability, and Risk of Metals. $2^{\mathrm{a}}$ ed. Springer-Verlag, New York.

AGUILAR, R., C. HORMAZÁBAL, H. GAETE \& A. NEAMAN, 2011. Spatial distribution of copper, organic matter and $\mathrm{pH}$ in agricultural soils affected by mining activities. Journal of Soil Science and Plant Nutrition, 11: 125-144.
ARANCIBIA, V., 2002. Ubicación geográfica, construcción, abandono y forestación de los tranques de relave de la V Región. Facultad de Recursos Naturales, Pontificia Universidad Católica de Valparaíso, Valparaíso, Memoria para óptar al título de Geógrafo, 139 pp. Inédita.

BADILLA-OHLBAUM, R.; R. GINOCCHIO, P. RODRÍGUEZ, A. CÉSPEDES, S. GONZÁLEZ, H. ALLEN \& G. LAGOS, 2001. Relationship between soil copper content and copper content of selected crop plants in central Chile. Envir. Toxicol. Chem., 12: 2749-2757.

BARREDO, J.I., 1996. Sistemas de información geográfica y evaluación multicriterio en la ordenación del territorio. Editorial RA-MA, Madrid, España.

COMISIÓN NACIONAL DE RIEGO, 2000. Cartografía digital de la V Región. [CD-ROM]. Comisión Nacional de Riego, Santiago. 
DE GREGORI, I.; E. FUENTES, M. ROJAS, H. PINOCHET, \& M. POTINGAUTIER, 2003. Monitoring of copper, arsenic and antimony levels in agricultural soils impacted and non-impacted by mining activities, from three regions in Chile. J. Environ. Monit., 2: 287-295.

\section{DIRECCIÓN GENERAL DE AGUAS,} 2004a. Diagnóstico y clasificación de los cursos y cuerpos de agua según objetivos de calidad. Cuenca del río Aconcagua. Dirección General de Aguas, Santiago. Disponible en http://www.sinia.cl/1292/ articles-31018_Aconcagua.pdf

DIRECCIÓN GENERAL DE AGUAS, 2004b. Evaluación de los recursos hídricos superficiales en la cuenca del río Aconcagua. Dirección General de Aguas, Santiago. http://www.dga.cl/ otros/informestecnicos/informestecnicos/ SDT165/INFACONCAGUAFINALWEB. pdf

\section{ENVIROMENTAL} SYSTEMS RESEARCH INSTITUTE, 1996. Using ArcView GIS software. ESRI Press, Redlands, CA.

FISHER, P. F., 1999. Models of uncertainty in spatial data. Geographical Information Systems, 1: 191-205.

FOLCHI, M., 2003. El beneficio del cobre por 'vía húmeda' y su impacto en el medio ambiente: Chile, 1904-1990. Simposio de Historia Ambiental Americana, 1418 de julio de 2003. Departamento de Ciencias Históricas, Facultad de Filosofía y Humanidades, Universidad de Chile, Santiago. http://www.historiaecologica.cl/ rmineria2.PDF

FOLCHI, M., 2006. Historia ambiental de las labores de beneficio en la minería de cobre en Chile, siglos XIX y XX. Tesis de doctorado, Departamento de Economía y de Historia Económica, Universidad Autónoma de Barcelona, Barcelona, 727 pp. Inédito.
GINOCCHIO, R.; G. CARVALLO, I. TORO, E. BUSTAMANTE, Y. SILVA \& N. SEPULVEDA, 2004. Micro-spatial variation of soil metal pollution and plant recruitment near a copper smelter in Central Chile. Environ Pollution, 3: 343-352.

GONZÁLEZ, S., 1991. Estrategias para la evaluación de impactos de procesos contaminantes en la agricultura. El conflicto minería-agricultura en Chile. Simiente, 1: 65-77.

GONZÁlEZ, S. \& R. ITE, 1992. Acumulación metálica en suelos del área bajo influencia de las chimeneas industriales de ventanas. Agricultura Técnica, 50: 214-219.

HUIDOBRO, G., 1861. Metalurgia del cobre en la provincia de Aconcagua. Anales de la Universidad de Chile, 19: 453-472.

INSTITUTO NACIONAL DE ESTADÍSTICAS, 2005. Chile: Ciudades, pueblos, aldeas y caseríos. Instituto Nacional de Estadísticas, Santiago. http:// www.ine.cl/canales/chile_estadistico/ demografia_y_vitales/demografia/pdf/ cdpubaldcasjunio2005.zip

LARA, L. \& L. ROMO, 2002. Atlas de Faenas Mineras. Minas y plantas de las regiones V, VI y XIII. Servicio Nacional de Geología y Minería, Santiago.

NEAMAN A., R. AGUILAR, C. HORMAZABAL, H. GAETE \& M. CISTERNAS, 2006. Distribución de la concentración de cobre en suelos agrícolas de la cuenca del Aconcagua. $57^{\circ}$ Congreso Agronómico de Chile, Santiago, 17-20 de octubre de 2006.

SÁNCHEZ, J. \& S. ENRÍQUEZ, 1996. Impacto ambiental de la pequeña y mediana minería en Chile. Departamento de Economía de la Universidad de Chile, Santiago. Inédito. Disponible en http:// www.bvsde.paho.org/bvsacd/cd27/ ambientechile.pdf 